A Iridjor purpuse UI li IE I ELillical Information Center is to provide the broadest dissemination possible of information contained in DOE.'s Research and Development Reports to business, industry, the academic community, and federal, state and local governments.

Although a small portion of this report is not reproducible, it is being made available to expedite the availability of information on the research discussed herein. 
LA-UR $-86-1333$

$00 N F-86077-2$

LA-UR-- 86-1333

DE86 010189

TITLE: INTRODUCTION TO STRING FIELD THEORY

AUTHOR(S): Joseph Lykken and Stuart Raby. Theoretical Division, T-8

Submitted TO: Proceedings of the Utah State Theoretical Phye Lco Workshop, Logan, Utah, February 24-28, 1986.

\section{DISCL.4IMER}

This roport was prepared as an accornt of work aponsored by an agency of the United Stules Government. Neither the United Stntes Government nor any agency thereof, nor any of their employees. makes any warranty, exprese or Implied, of asumes any logal itability of reanonajbillty for the eccurncy, cumpletereas, or usofulnew of any information, apparntus, product, of proceses dieclosed, or represents that lis use would not infringe privately owned rights. Refor. ence herrin 10 any apecille commerctal product, proceses, or cervitos by trade name. Ifademark. manufueturer, or otherwise does not neccusarlly constitute or imply its endorsoment, recominendation, of favoring by the United States Covernment of any aganev thoreof. Tho viewa

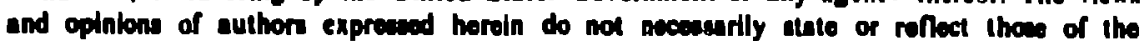
United Statee Government of any asency thoreof.

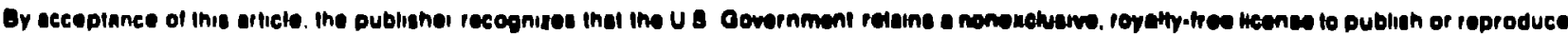

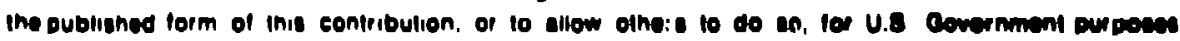




\title{
Introduction to String field Theory
}

\author{
Joseph Lykken \\ and \\ Stuart Raby* \\ Theoretical Division T-8 \\ Mail St.op B285 \\ Los Alamos National Laboratory \\ Los Alamos, New Mexico 87545
}

\begin{abstract}
We propose an action for an interacting closed bosonic string. Our formalism relies heavily on ideas discussed by Witten for the open bosonic string. We also obtain the gauge flxed quantum action fur the fully interacting open bosonic string.

* Lectures given at Utah State Urilversity, Logan, Utah, February 24-28, 1986.
\end{abstract}




\section{Introduction}

String theories may very well provide a unified description of all the known paiticles and their interactions- including gravity. The 17 or more phenomenological parameters of the standard model may. in principle, be determined in this fundamental theory with no free parameters. Such are the fervent hopes of particle theorists. Yet if we are in understand this new theory we must relinquish the paradigms of the point particle, local field theory and local gauge symmetries. These concepts are presumably relevant descriptions of nature only in a low energy approximation. For example, the principle of general coordinate Invarlance is probably not a useful concept as applles to the string. The Virasoro-Shapiro model contains a massless spin two "graviton". However this state has no loca! four point interactions as is the case for the graviton in general relativity.' Thus the "graviton" of the Virasoro-Shapiro model is not a simple piece of a tensor under general coordinate transformations. ${ }^{2}$ A new symmetry principle lased on the string is thus needed to supercede some old but valued contepts at olstance scales where stringiness becomes relevant.

Recent work towards a covarlant string rlelo theory $[1-27)$ is partly motivated by this desire to rimi the symmetry principle behind the theory. We feel that a significant step in this direction has recently been taken by Witten (3) in his creation of an interacting cpen bosonic string field theory using some loeas of Cornes [29] on nor-cornmutative differential geometry. In this paper we explore these ideas with respect 
to the open bosonic string in some rore detail. Using the intuition so obtained we propose an action for in interacting closed bosonic string rield theory.

In section 2 we review Witten's theory of the open Dosonic string. We use a notation, however, more akin to that of Siegel and 2 wiebach [2]. We present the gauge fixed BRST [30-34] invariant action for Witten's theory. This result can be round in equation (1.33). In section 3 we describe our results on the closed bosonic string. We discuss some new axioms which can lead to a gauge invariant interacting closed bosonic string action. The relevant equations are $(2.20)-(2.25)$. We then motivate the new set of axioms. In section 4 we summarize our results and discuss the problems which remain to be explored.

\section{The Open Bosonic String}

Following Siegel $[1]$ we derine a real string functional

$$
\phi\left(x_{\mu}(\sigma), c(\sigma), \tilde{c}(\sigma)\right) \text {. }
$$

and a BRST generator $Q$, satisfying $Q^{2}=0[35,36]$, represented as a differential operator acting on --

$$
\left.Q=\sqrt{\pi \alpha^{\prime}} \int_{-\pi}^{T} d \sigma \dot{c}(\sigma) \mid 1 / 2 \hat{p}^{2}(\sigma)-1 / \alpha^{\prime} \dot{c}^{\prime}(\sigma) \Pi_{c}(\sigma)\right)
$$

where

$$
\begin{aligned}
& \dot{p}_{\mu}(\sigma)=1 / \sqrt{2}\left[\delta / \delta \times \mu(\sigma)-1 / \alpha^{\prime} x_{\mu}^{\prime}(\sigma)\right] . \\
& \dot{c}(\sigma)=\alpha^{\prime} / \sqrt{2}\left[\delta / \delta c(\sigma)+1 / \alpha^{\prime} \tilde{c}(\sigma)\right] . \\
& \left.\Pi_{c}(\sigma)=1 / \sqrt{2} \mid \delta / \delta \tilde{c}(\sigma)+1 / \alpha^{\prime} c(\sigma)\right] .
\end{aligned}
$$


The commuting (anti-commuting) coordinates $x_{\mu}(\sigma)(c(\sigma)$. $\tilde{c}(\sigma))$ with $\mu=1, \cdots, 26$ and $0 \leqslant \sigma \leqslant \pi$ satisfy the boundary conditions $x_{\mu}^{\prime}(\sigma)$ $z x_{\mu}(\sigma) / d \sigma=0, c^{\prime}(\sigma)=0, \tilde{c}(\sigma)=0$ at $\sigma=0, \pi$. (The coordinates for the region $-\pi \leqslant 0 \leqslant 0$ are then obtained by analytic continuation. $\alpha$ is the string terision.)

The string functional can be expanded in terms of an inf inite rumber of local component fields. This expansion is particularly useful when making contact with local field theory. We define the differential operators $a_{n \mu} \cdot B_{n} \cdot B_{n}$ for $-\infty \leqslant n \leqslant \infty$ as the normal modes of the operators given in (eq. 1.3)-

$$
\begin{aligned}
& \beta_{\mu}(\sigma)=1 / \sqrt{2 \pi \alpha^{\prime}} \sum a_{n \mu} e^{-i n \sigma} . \\
& \dot{c}(\sigma)=\sqrt{\alpha^{\prime} / 2 \pi \sum} B_{n} e^{-i n \sigma} . \\
& \Pi_{c}(\sigma)=1 / \sqrt{2 \pi \alpha^{\prime}} \sum \beta_{n} e^{-i n \sigma} .
\end{aligned}
$$

They satisfy the canonical commutation and anti-commutation relations

$$
\begin{aligned}
& :\left(a_{n \mu} \cdot a_{m \nu}\right)=n, \delta_{n+m, 0} \eta_{\mu \nu} . \\
& \left\{B_{n} \cdot B_{m}\right\}=\delta_{n<m, 0} .
\end{aligned}
$$

We also define a grourd state functional $\phi_{0}$ satisfying

$$
a_{n \mu} \Phi_{0}=B_{n} \Phi_{0}=\tilde{B}_{m} \Phi_{0}=0 \text { for } n \geq 0, n 1>0 \text {. }
$$

The component expression is then given by

$\phi=\left(i \tilde{B}_{1}^{\dagger} B(x)+\cdots+c\left(\varphi(x)+a_{1 \mu}^{\dagger} A^{\mu}(x)+B_{1}^{\dagger} \bar{\eta}(x)+\tilde{B}_{1}^{\dagger} \eta(x)+\cdots\right)\right) \varphi_{0}$

where $c=\sqrt{\alpha^{\prime} / \pi} \beta_{0}$ and $\partial_{c}=\sqrt{\pi / \alpha^{\prime}} B_{0}$. 
$\checkmark$ is a commuting field. Thus the rields $\varphi, A^{\mu}, B$ are also commuting and correspand to the tachyon, abelian gauge field and Lautrup-Nakanishi auxiliary field. $\eta$ and $\bar{\eta}$ are the anti-commuting Fadeev-Popor ghost and anti-ghost fields cornected with $A^{\mu}$ and $B$.

A global BRST trans? ormation of $\$$ is given by

$$
\delta \Phi=i \in Q \Phi
$$

which leaves the free action

$$
S_{0}=\int \Phi^{\top}[0, \rho] \phi
$$

invariant. $\left(\Phi^{\top} \equiv \Phi\left[x_{\mu}(\pi-\sigma), c(\pi-\sigma),-\tilde{c}(\pi-\sigma)\right]\right) \rho$ is the gauge fixing operator and the Feynman-Siegel gauge is obtained by the choice

$$
\rho=\left\{c, \partial_{c}\right\} \text {. }
$$

A particularly simple subspace to consider, for lllustrative purposes, is that including the massless sector alone i.e., $A^{\mu}, B, \eta$ and $\bar{\eta}$. In this subspace the BRST generator $Q$ assumes the form

$$
Q_{0}=\left(\beta_{1} a_{1}^{+} \cdot i \partial+\beta_{1}^{\dagger} a_{1} \cdot i \partial-\partial_{c} \square-\beta_{1}^{\dagger} \beta_{1} c\right)
$$

and satisfies $Q_{0}{ }^{2}=0$ ( we have taken $\alpha^{\prime}=2 \pi$ ). It is then trivial to check that the BRST transformation (eq. 1.8) for the massless fields is given by

$$
\begin{aligned}
& \delta A^{\mu}=-\epsilon \partial^{\mu} \eta \\
& \delta \eta=0 \\
& \delta B=\epsilon \square \eta \\
& \delta \bar{\eta}=\epsilon(\partial \cdot A+B) .
\end{aligned}
$$

Let us now consider the local gauge invariant theory of Witten. We must first define the physical subspace of $\$$. Following witten we 
consider the ghost number operator given by

$$
N_{g}=1 /{ }_{2}\left[\partial_{c}, c\right]+\sum_{n>0}\left(\beta_{n}^{\top} B_{n}-B_{n}^{T} \beta_{n}\right) \text {. }
$$

Then

$$
N_{g} \Phi_{0}=1 / 2 \Phi_{0}
$$

and the physical sector is derined to have ghost number $-1 / 2$. (We shall denote by capital Lat in letters string functionals satisfying the constraint

$$
N_{g} A=-1 / 2 A \text {.) The component expansion is given by }
$$

$$
A=\left\{i B_{i}^{\dagger} B(x)+\cdots+C\left(\varphi(x)+a_{1 \mu}^{\dagger} A \mu(x)+\cdots\right)\right\} \Phi_{0} .
$$

The free field equations are then given by

$$
Q A=0
$$

which is invariant under the local gauge transformation

$$
\delta A=Q E \text {. }
$$

Note that $\left[N_{Q}, Q\right]=Q$ and thus $\&$ must have ghost number $-3 / 2$. In the massless subsector we find eqs. 1.15 and 1.16 imply

$$
\begin{aligned}
& \square A_{\mu}+\partial_{\mu} B=0, \quad \partial \cdot A+B=0 \\
& \delta A_{\mu}=\partial_{\mu} \lambda, \delta B=-\square \lambda
\end{aligned}
$$

where " $\epsilon=\left(\mid \subset \boldsymbol{B}_{1}^{+} \lambda(x)+\cdots\right) \Phi_{D}$. Eqs. 1.17 are equivalent to the free Maxwell equations $a^{\mu} F_{\mu \nu}=0$. The free action $S_{W O}$ is olven by

$$
S_{W O}=\int A^{T} Q A \text {. }
$$

In order to generalize this theory to include interacilons witten relies on the axioms of the non-commutative differential gecmetry of connes. In the rest of this section we shall

1) review these axioms, and

2) discuss an explicit representation of them 


\section{Abstract Axioms}

We want the string functionals A to satisfy propertles analogous to inose of forms in differential geometry and for the BRST generator $Q$ to act as a derivation. $Q$ already satisfies the first axiom

i) $\mathbf{a}^{2}=0$.

We define a froduct rule denoted by * and an integral $\int$ which satisfy:

ii) associativity or "product-

$$
(A * B) * C=A *(B * C) \text {. }
$$

Although the product is non-commutative ( $A^{*} B=B^{*} A$ ). we demand

iii) $\int^{*} B=(-1)^{A B} \int^{*} A$

where $(-1)^{A B}=(-1$, If $A$ and $B$ are Grassman odd: +1 , otherwise).

The additional properties of a derivation are

iv) $\int \mathbf{Q} X=\mathbf{0}$ for any string functional $X$ and. $Q$ must satisfy

v) the Leibnitz rule-

$$
Q(A \sqcap B)=Q A \sqcap B+(-1)^{A} A * Q B
$$

where $(-1)^{A}=(-1$, if $A$ is Grassman odd: +1 . otherwise $)$.

Given these properties we can then define the gauge flelc strength

$$
F=Q A \cdot A \times A
$$

which under the local gauge transformation

$$
\delta A=Q E+[A, E]
$$

(where $\left.[A, \epsilon]=A^{m} \in-\epsilon^{*} A\right)$ transforms by

$$
\delta F=[F, \in] \text {. }
$$

I Note that the group structure implicit in this abstract algebra is encoded in the " product. For example it is sasy to see that the 
transformation of $A$ defined in eq. 1.20 satisfies the group property

$$
\left[\delta_{2}, \delta_{1}\right] A=\delta_{3} A
$$

where the infinitesimal gauge functional parameters $\epsilon_{\mathcal{l}}, i=1,2,3$ are related by $\left.\epsilon_{3}=\left[\epsilon_{2}, \epsilon_{1}\right].\right\}$ Finally the action for the interacting theory is given by

$$
S_{W}=\int\left(A^{*} Q A+2 / 3 A^{*} A^{*} A\right)
$$

which is invariant under (1.20). The equations of motion are given by

$$
\delta 5 / \delta A=F=0 \text {. }
$$

The ghost numbers of $\int$ and * were determined by witten to be $-3 / 2$ and $+3 / 2$, respectively. Moreover $\int X=0$ unless $X$ has ghost number $+3 / 2$. Hence a non-trivial action has net ghost number zero.

\section{Bepresentation of the Axloms}

Witten has shown that an associative " product requires treating the point $\sigma=\pi / 2$ special. Thus $A^{*} B$ is defined by a process of "sewing" the right half of string $A$ with the left half of string $B$ as depicted in rig.1. This statement will be made more precise shortly. If $A^{\text {w }} B$ is given by fig.l, then integral $f$ might be expected to "sew" the remaining halves of the string to each other ,as in fig.2. In this way we obtain the "sewing condition"

$$
\int A^{\top} B(\text { eq. } 1.18)=\int A \wedge B
$$

where $A^{\top}=A\left[x_{\mu}(\pi-\sigma), c(\pi-\sigma),-\tilde{c}(\pi-\sigma)\right]$.

\section{Ihe Intearal - 1}

We shall now obtain an explicit representation of $\int$. The two main 
9

properties it must satisfy are Axiom (iv) and ghost number $-3 / 2$. The "naive" integral is represented by

$$
\begin{aligned}
\int=\int & \text { where } D=\prod_{\sigma=0}^{\pi} d x_{\mu}(\sigma) d c(\sigma) d \tilde{c}(\sigma), \delta=\prod_{\sigma=0}^{\pi / 2} \delta\left(x^{0}\right) \delta\left(c^{0}\right) \delta\left(c^{e}\right) \text { and } \\
x_{\mu}{ }^{0}(\sigma) & =x_{\mu}(\sigma)-x_{\mu}(\pi-\sigma) \\
c^{0}(\sigma) & \equiv c(\sigma)-c(\pi-\sigma) \\
\tilde{c}^{2}(\sigma) & \equiv \tilde{c}(\sigma) \cdots \tilde{c}(\pi-\sigma) .
\end{aligned}
$$

The ghost number of the factors are $D[1 / 2], \delta[0]$. Thus the "naive" integral has the wrong ghost number. Moreover we find that $\int D \delta Q X=0$ in general, due to complications associated with the special point $\sigma=\pi / 2$. Thus an insertion is needed. The unlque integral with ghost number $-3 / 2$ and satisfying Axiom (iv) is given by

$$
\int=\int D \delta 1
$$

where $1=c(\pi / 2) \delta / \delta \tilde{c}(\pi / 2)$. We then rind $\int Q x=0$ only in 26 dimensions.

\section{The product}

Thie most difficult constraint the * product must satisfy is the Leibnitz rule. Recall that the BRST charge $Q$ (eq.1.2) contains products of up to three derivatives. A "naive" definition of the "product is given by the expression ( see fig.1)

$$
\begin{aligned}
& A^{*} B=\int \prod_{\sigma=\frac{x}{2}}^{\pi} d x^{\prime} \mu(\sigma) d c^{\prime}(\sigma) d \tilde{c}^{\prime}(\sigma) \prod_{\sigma_{0}}^{\pi / 2} d x^{2} \mu(\sigma) d c^{2}(\sigma) d \tilde{c}^{2}(\sigma) \\
& \times \prod_{0}^{2} \delta\left(x^{2}(\sigma)-x^{\prime}(\pi-\sigma)\right) \delta\left(c^{2}(\sigma)-c^{1}(\pi-\sigma)\right) \delta\left(\tilde{c}^{2}(\sigma)+\tilde{c}^{1}(\pi-\sigma)\right) A[1] B(2] \\
& \text { - } \int \mu^{*} A B
\end{aligned}
$$


( where we use the shorthand notation $A[1]=A\left[x_{\mu}^{\prime}(\sigma), c^{\prime}(\sigma), \tilde{c}^{\prime}(\sigma)\right]$ ). Since $Q$ is defined by an integral over a local function of $\sigma$, it is possible to derine a new charge $Q^{\prime}$ with the point $\sigma=\pi / 2$ removed. It is trivial to show that $Q$ " satisfies a Lelbnitz rule on the "naive" " product. Consider $\left.Q^{\prime}\left(A^{*} B\right)=\int \mu^{*} \mid\left(Q_{-}^{\prime} A\right) B+(-1)^{A} A\left(Q^{\prime}{ }_{+} B\right)\right]$ where $Q_{ \pm}^{\prime}$ are defined by integrals over the \pm sectors of strings $A$ or $B$ ( see fig. 1). However, using the following identity, which is a consequence of integration by parts, $\int \mu^{*}\left[\left(Q^{\prime}{ }_{+} A\right) B+(-1)^{A} A\left(Q^{*}-B\right)\right]=0$ and

$Q^{\prime} A \equiv\left(Q_{+}^{\prime}+Q_{-}^{\prime}\right) A$ we find $Q^{\prime}\left(A^{n} B\right)=O^{\prime} A^{n} B+(-1)^{A} A^{n} Q^{\prime} B$. The difficulty in satisfying the Leibnitz rule is thus all contained in the treatment at the special point $\sigma=\pi / 2$.

We have not yet solved this difficult problem. Nevertheless we may state some general properties the * product must satisfy in a notation which will be useful later in our discussion of the closad string. In general we may define a "product by the expression

$$
A^{*} B[3]=\int D_{1} D_{2} K[312] A[1] B[2]
$$

where $D_{i} \equiv \prod_{\sigma=0}^{\pi} d x_{\mu}^{i}(\sigma) d c^{i}(\sigma) \sigma^{-1}(\sigma)$ and the Kernel $K$ must satisfy

(a) "sewing condition" (eq.1.25)-- $\int \mathrm{D}_{3} \delta_{3} / 3 \mathrm{~K}[312]=8[12] ;(1.3 / a)$

(b) Leibnitz rule $-\sum_{i=1}^{3} a_{i} k[312]=0$, and

(c) associativity - $\int D_{3} K[534] \mathrm{K}[312]=-\int D_{3} K(513] \mathrm{K}(324) .(1.31 \mathrm{C})$

Propert!! (a) fixes the ghost number of $K$ to be $+1 / 2$ for the open string and hence the ghost rumber of the " product is $+3 / 2$. It can also be used to show that $K$ is Grassman even, using $D_{3}$ (odd), $\delta_{3}$ [even), $I_{3}$ [even] and $8[12]$ lodd). 
Once the "product ( $\alpha$ the Kernel $k$ ) is determined we may then obtain the interacting field equations in component form. Work in this direction is still in progress. Tnroughout the remainder of this section we shall assume that there exists a $K$ satisfying $(a-c)$.

The " product is also significant on a deeper level. It defines a sort of matrix multiplication where the string functional A ( a cornection un some fibre bund!e) can be thought of as an infirite dimensional matrix. Gauge transformations $\delta A(1.20)$, as usuai, describe the motion of $A$ along the fiure. However the base manifold of this fibre bundle is trivial; it's just a poirit.

We end this section with a discussion of the gauge fixed quantum action for the fully interacting theory. Consider first the free fielo case. Witten's gauge invariant action $S_{W O}=\int A^{*} Q A$ is invariant under $\delta A=06 . \quad A$ has ghost number $-1 / 2$. The quantum string functional ( where by "quantum" we mean it includes the BRST ghost and anti-ghost fields which are necessary for a quantum description of the theory ) $\square=A+\equiv$ where the field $\equiv$ includes all ghost numbers, excluding $-1 / 2$. Siegel's gauge fixed action may then be rewritten using the notation of $\int$ and der ined previously. We have

$$
\begin{aligned}
S_{0} & =\int Q^{*}[Q, p] \Phi \\
& \left.=\int A^{N} Q A+\int A^{N}(\mid Q, p)-Q\right) A+\int \Xi^{N}(Q, p) \equiv
\end{aligned}
$$

where the three terms may be identified as the gauge invariant action, the gauge fixing term and the Fadeev-Popov ghost actir. respectively.

$S_{0}$ is Invariant under global BRST transformations $\delta \phi=i \leqslant 0 \phi$. Note the physical sector transforms according to $\delta A=Q(-1 f E \mid-3 / 2)$. 
12

BRST Invariant Action

Let us now generalize Siegel's free gauge fixed quantum action to the fully interacting theory. We contend that

$$
S_{q u}=\int\left(\phi^{*}[Q, \rho] \phi+2 p \phi * \phi^{*} \phi\right)
$$

is invariant under the nonlinear global BRST transformation

$$
\delta \Phi=i \in\left(Q \phi+\phi^{*} \phi\right)
$$

satisfying $\delta^{2}=0$. The physical sector obeys

$$
\delta A=Q Q+\left[A,[Z]+i \varepsilon \Xi_{n_{1}}{ }^{*} \Xi_{n_{2}} \ln n_{1}+n_{2}=-2\right.
$$

where $\xi=-i \epsilon \equiv \mid-3 / 2$. Except for the last term, this is just a generalization of global BRST transformations for non-abelian gauge theories.

3. The Closed Bosonic String

Following siegel (once more) we define a real closed string functional

$$
\phi\left[x_{\mu}(\sigma), c(\sigma), \tilde{c}(\sigma)\right]
$$

ind a BAST generator $Q$, satisfying $Q^{2}=0$. $Q$ is given by

$$
\begin{aligned}
Q=\sqrt{2 \pi \alpha^{\prime}} & \int_{-\pi}^{\pi} d \sigma\left(\hat{c}(\sigma)\left|1 / 2 \hat{P}^{2}(\sigma)-1 / \alpha^{\prime} \hat{c}^{\prime}(\sigma) \pi_{c}(\sigma)\right|(2.2)\right. \\
& \left.\left.+\overline{\hat{c}(\sigma)} \mid 1 / 2 \bar{\beta}^{2}(\sigma)+1 / \alpha^{\prime} \bar{c}^{\prime}(\sigma) \bar{\pi}_{\hat{c}}(\sigma)\right)\right\}
\end{aligned}
$$

where

$$
\begin{aligned}
& \left.\hat{p}_{\mu}(\sigma)=1 / \sqrt{2} \mid \delta / \delta \times \mu(\sigma)-1 / \alpha^{\prime} x_{\mu}(\sigma)\right] . \\
& \left.\hat{c}(\sigma)=\alpha^{\prime} / \sqrt{2} \mid \delta / \delta c(\sigma)+1 / \alpha^{\prime} \tilde{c}(\sigma)\right) . \\
& \left.\Pi_{c}(\sigma)=1 / \sqrt{2} \mid \delta / \delta \tilde{c}(\sigma)+1 / \alpha^{\prime} c(\sigma)\right)
\end{aligned}
$$




$$
\begin{aligned}
& \overline{\hat{P}}_{\mu}(\sigma)=1 / \sqrt{2}\left[i \delta / \delta x^{\mu}(\sigma)+1 / \alpha^{\prime} x_{\mu}(\sigma)\right] . \\
& \overline{\hat{c}}(\sigma)=\alpha^{\prime} / \sqrt{2}\left[\delta / \delta c(\sigma)-1 / \alpha^{\prime} \tilde{c}(\sigma)\right] . \\
& \bar{\pi}_{c}(\sigma)=1 / \sqrt{2}\left[-\delta / \delta \tilde{c}(\sigma)+1 / \alpha^{\prime} c(\sigma)\right] .
\end{aligned}
$$

The commuting (anti-commuting) coordinates $x_{\mu}(\sigma)(c(\sigma), \tilde{c}(\sigma))$ with $\mu=1, \cdots, 26$ and $-\pi \leqslant \sigma \leqslant \pi$ satisfy the periodic boundary conditions $x_{\mu}(\sigma)=x_{\mu}(\sigma+2 \pi), c(\sigma)=c(\sigma+2 \pi), \tilde{c}(\sigma)=\tilde{c}(\sigma+2 \pi)$. Note that $\tilde{c}(\sigma)$ now has a zero mode, unlike the case of the open string.

As in the case of the open string, we may expand in terms of an infinite number of local component fields. We define the differential operators $a_{n \mu}, B_{n}, \bar{B}_{n}, \bar{s}_{n \mu}, \bar{B}_{n}, \bar{B}_{n}$ for $\cdots \infty \leqslant n \leqslant \infty$ by

$$
\begin{aligned}
& \hat{p}_{\mu}(\sigma)=1 / \sqrt{2 \pi \alpha^{\prime}} \sum a_{n \mu} e^{-i n \sigma} . \\
& \dot{c}(\sigma)=\sqrt{\alpha^{\prime} / 2 \pi} \sum \beta_{n} e^{-i n \sigma} . \\
& \Pi_{c}(\sigma)=1 / \sqrt{2 \pi \alpha^{\prime}} \sum \beta_{n} e^{-i n \sigma} . \\
& \bar{p}_{\mu}(\sigma)=1 / \sqrt{2 \pi \alpha} \sum \bar{\delta}_{n \mu} e^{i n \sigma} . \\
& \bar{c}_{\hat{c}(\sigma)}=\sqrt{\alpha} / 2 \pi \sum B_{n} e^{i n \sigma} . \\
& \bar{\pi}_{c}(\sigma)=1 / \sqrt{2 \pi \alpha^{\prime}} \sum \bar{B}_{n} e^{i n \sigma}
\end{aligned}
$$

satisfying the canonical commutation and anti-commutation relations

$$
\begin{aligned}
& \left(a_{n \mu}, a_{m \nu}\right)=n \delta_{n+m, 0} \eta_{\mu \nu}, \\
& \left(\beta_{n}, \delta_{m}\right)=\delta_{n+m, 0} . \\
& \left(\bar{\delta}_{n \mu}, \bar{\delta}_{m \nu}\right)=n \delta_{n+m, 0} \eta_{\mu \nu} . \\
& \left(\nabla_{n}, \bar{\delta}_{m}\right)=\delta_{n+m, 0}
\end{aligned}
$$


with all others zero. We also derine a ground state functional $\Phi_{0}$ satisfying

$$
\begin{aligned}
& a_{n \mu} \Phi_{0}=B_{m} \oplus_{0}=\bar{B}_{m} \Phi_{0}=\bar{a}_{n \mu} \Phi_{0}=B_{m} \oplus_{0}=\bar{B}_{m} \oplus_{0}=0 \\
& \text { for } n \geq 0, m>0 .
\end{aligned}
$$

We also have

$$
\partial_{c} \Phi_{0}=\partial_{c} \Phi_{0}=0
$$

where $\partial_{c}=\sqrt{\pi / \alpha^{\prime}}\left(B_{0}+B_{0}\right)$ anc $\partial_{c}=\sqrt{\pi / \alpha^{\prime}}\left(B_{0}-\bar{B}_{0}\right)$.

The component expansion of includes a tachyon, massless graviton, Kalb-Ramond anti-symmetric tensor, dilaton and an infinite number of massive fields. It also includes the necessary Fadeev-Popor ghosts, auxiliary fielos and many more unphysical fields.

Global BRST trionsformations are again given by

$$
\Delta Q=i \in Q \Phi \text {. }
$$

We now want to discuss a gauge invariant description for the closed bosonic string. The physical sector of may be defined a la Witten by lits ghost number content. We define the ghost number operator

$$
N_{g}=1 / 2\left(\partial_{c}, c\right)-1 / 2\left(\partial_{\tilde{c}}, \tilde{C}\right) \cdot \sum_{n>0}\left(B_{n}^{\dagger} \tilde{B}_{n}-\tilde{B}_{n}^{\dagger} B_{n}+B_{n}^{\dagger} \tilde{B}_{n}-\tilde{B}_{n}^{\dagger} B_{n}\right)
$$

with

$$
N_{g} \Phi_{0}=0 \text {. }
$$

Then the physical sector la definad to have ghost number -1 . (We shall use capltal Latin letters for physical string functionals which satisfy $N_{\theta} A=-A$.) We shall impose two additional constraints on in order to define the physical sector. The first is the standard requirement that the string functional is invarlant under constant reparametrizations of o 
i.e. the "origin" of the string has no physical significance, or

$$
A\left[x_{\mu}(\sigma+1), c(\sigma+1), \tilde{c}(\sigma+1)\right]=A\left[x_{\mu}(\sigma), c(\sigma), \tilde{c}(\sigma)\right\} \quad(2,10)
$$

for constant shift $f$. Finally we constrain the dependence of $A$ on the ghost zero mode $\tilde{c}$. We dernano that $A$ be expressible in the form $A=\partial_{\tilde{c}} X$ which implies $\quad \partial_{\tilde{c}} A=0$. To summarize, the physical sector of $\$$ is defined to satisfy

$$
N_{g} A=-A, \triangle N A=0, A=\partial_{\tilde{c}} X
$$

where $\quad \Delta N=N-N$ (the number of right minus left movers of the string) is the generator of censtant $\sigma$ reparametrizations.

The free field equations of motion are given by

$$
Q A=0
$$

with the corresponding gauge invar iance

$$
\delta A=Q \epsilon \text {. }
$$

The component expansion of $A$ is given th'

$$
\begin{aligned}
& A=\left(i \bar{B}_{1}^{\dagger} \bar{\partial}_{1 \mu}^{T} \bar{B} \mu(x)+1 \bar{B}_{1}^{\dagger} a_{1 \mu}^{r} B \mu(x)+\cdots+c(\Phi(x)+\right. \\
& \left.\left.\bar{\partial}_{1 \mu}^{\dagger} a_{1 \nu}^{\dagger} n^{j \nu}(x)+B_{1}^{\dagger} \bar{B}_{1}^{\dagger} \bar{X}(x)+\bar{B}_{1}^{\dagger} B_{1}^{\dagger} X(x)+\cdots\right)\right) \nabla_{0} \text {. }
\end{aligned}
$$

In the massless subspace the BRST generator takes the simple form

$$
\begin{aligned}
O_{0}=\left(B_{1} \partial_{1}^{\dagger} \cdot 1 \partial+B_{1}^{\dagger} \partial_{1} \cdot 1 \partial+B_{1} \delta_{1}^{\dagger} \cdot 1 \partial \cdot B_{1}^{\top} \delta_{1} \cdot 1 a-\partial_{C} D\right. \\
\left.-\left(B_{1}^{\dagger} B_{1}+B_{1}^{\dagger} B_{1}\right) C\right)
\end{aligned}
$$

satisfying $Q_{0}{ }^{2}=0$ (with $\alpha^{\prime}=4 \pi$ ). It is then trivial to check that in the massless subsector eqs. 2.12 and 213 give the equations of motion 
$\square n^{\mu \nu}+\partial^{v} B^{\mu}+\partial^{\mu} B^{v}=0$,

$\square X-\partial \cdot B=0, \square \bar{X} \cdot \partial \cdot \bar{B}=0$

$\partial_{v} \hat{h}^{\mu \nu}+\bar{B}^{\mu}+\partial^{\mu} x=0$,

$\partial_{\mu} n^{\mu v}+B^{v}-\partial^{v} \bar{X}=0$;

and local gauge transformations

$$
\begin{aligned}
& \delta n^{\mu \nu}=\partial \tau^{\mu}+\partial^{\mu} \xi^{\nu} \\
& \delta \bar{\varepsilon}^{\mu}=-\square \xi^{\mu}+\partial^{\mu} \eta . \delta B^{\nu}=-\square \xi^{\nu}-\partial^{\nu} \eta \\
& \delta \bar{X}=\partial \cdot \tau-\eta, \delta X=-\partial \cdot \xi-\eta
\end{aligned}
$$

where $\epsilon=\left\{\bar{B}_{1}^{\dagger} \tilde{B}_{i}^{\dagger} \eta(x)+\left\{c\left(\tilde{B}_{1}^{\dagger} \bar{\partial}_{1 \mu}^{\dagger} \tau^{\mu}(x)+\overline{\mathbb{B}}_{1}^{\dagger} a_{1 \mu}^{\dagger} \varepsilon^{\mu}(x)\right)+\cdots\right\} \oplus_{0}\right.$.

These are just the equations of motion for linearized gravity, the Kald-Ramond gauge field and a massless dilaton.

An action may be constructed for the free theory. We find ${ }^{3}$

$$
S_{0}=\int A^{T} y[\hat{c}, 0] A
$$

where $A^{\top}=A\left[x_{\mu}(-\sigma), c(-\sigma),-\tilde{c}(-\sigma)\right]$. Note the insertion of the ghost zero mode coordinate $\tilde{c}$. This is necessary; otherwise the integral would vanish upon $\tilde{c}$ integration. The equations of motion derived from this action are

$$
\partial \tilde{c}|\tilde{c}, Q| A=0
$$

which (using the constraints eq. (2.11)) is equivalent to eq. (2.12). (Note that $Q$ may generally be written in the form $Q=Q^{\prime}+\sqrt{\pi / \alpha^{\prime}} \Delta N \tilde{c}$ $\cdot \sqrt{\alpha^{T} / \pi} \Delta T \partial_{\tilde{c}}$ where we have made the $\tilde{c}$ dependence explicit. It is then trivial to prove the userul identity $Q A=1 / 2 \partial_{\tilde{c}}[\tilde{c}, Q / A$. 
Let us now discuss our proposal for the fully interacting closed bosonic string theory. We want to write an action which is similar to that for the open bosonic string (eq. 1.23). In order to accomplish this we must change some of the axioms of section 2 . We shall assume that we can define an integral $\int$ and " product which satisfy the following axioms. Although we have not yet completely defined a representation for the new axioms, we shall try to motivate each one by comparison with our knowledge of the open string. It is not inconceivable to us that the axioms we discuss may, in fact, already be represented by the path integral expressions defined by Witten.

\section{Abstract Axioms}

$$
\therefore \mathbf{Q}^{2}=\mathbf{0}
$$

We def ine a product rule which is Grassman odd and satisf ies $\triangle N\left(A^{*} B\right)=0$ if $\triangle N A=\triangle N B=0$, but $\partial_{\tilde{c}} A^{\prime \prime} B=0$, even if $\partial_{\tilde{c} A}=\partial \tilde{c} B=0$. The "associativity" axioms of the "product are

ii) "sssociativity"-

$$
\begin{aligned}
& \left(A^{*} B\right)^{\star} C=-(-1)^{A} A^{*}\left(B^{*} C\right) \\
& \partial_{\tilde{c}}\left(\partial_{\tilde{c}}\left(A^{n} B\right)^{n} C\right)=\partial_{\tilde{c}}\left(A^{n} \partial_{\tilde{c}}\left(B^{n} C\right)\right) \text {. }
\end{aligned}
$$

The integral is defined to satisfy

iii) $\int^{M} B=-(-1)^{A B} \int B^{N} A$.

$Q$ satisfies the additional axioms

iv) $\int Q X=0$ for any string functional $X$, and

v) the "Lelbnitz rulo"-

$$
-Q(A \cdots B)=Q A \backsim B+(-1)^{A} A \sqcap Q B \text {. }
$$


18

Note that the axioms (i) and (iv) are identical to those of the open string. (ii),(iii) and ( $v$ ) have been altered. We shall also assume that the integral $f$ and " product can be defined to have ghost number -3 and +3 , respectively.

Before we attempt to justify our apparently bizarre set of axioms, let us first discuss some results which follow from their use. We are able to def tine a field strength

$$
F=\partial \tilde{c}\left(1 / 2[\tilde{c}, Q] A+A^{*} A\right)=Q A+\partial \tilde{c}\left(A^{*} A\right)
$$

which under the local gauge transformation

$$
\delta A=Q \epsilon+\partial \tilde{c}(A, \epsilon)
$$

(where $[A, \epsilon]=A^{*} \epsilon-\epsilon^{N} A$ ) transforms by

$$
\delta F=\partial \tilde{c}[F, \epsilon] \text {. }
$$

The fully interacting theory is then defined by the action

$$
S=\int\left(A^{*} 1 / 2[\check{c}, Q] A+2 / 3 A^{*} A^{*} A\right) \text {. }
$$

Note trial as a result of the axioms $\int\left(A^{*} A\right)^{*} A=\int A^{*}\left(A^{*} A\right)$.I it is invariant under the transformation (2.21). A proof of this statement requires using the following identities which follow from integration by parts and the "sewing condition":

-sewing conation" - $\int A^{n} B=\int A^{\top} B$

(where $A^{\top}$ is defined after eq.(2.18))

identities -

$\int(\partial \tilde{c} A)^{*} B=(-1)^{A} \int A^{*} \partial \tilde{c} B$

$\int A *|\tilde{c}, Q| B=\int((\tilde{c}, Q] A)^{M B}$.

The equations of motion are given by 


$$
\delta S / \delta X=F=0
$$

where $A n \partial \tilde{c} \tilde{x}$. For the free theory these equations reduce to those of eq.(2.12) which has been shown to include the equations of linearized gravity.

\section{Bepresentation of ine Axioms}

\section{The integral - I}

Our definition of ne integral for the closed string is a simple generalization of our results for the open string. The "naive" integral is defined by folding the closed loop in half (see fig. 3) and "sewing" the two halves together. There are now two singular points at $\sigma=0$ and $\pi$. which requires an insertion in order to satisfy axiom (iv). The correct measure is given by

$$
\int=\int D \delta 1
$$

where $\quad D=\pi_{\sigma-\pi} d x_{\mu}(\sigma) d c(\sigma) d \tilde{c}(\sigma), \delta=\prod_{\pi=0}^{\pi} \delta\left(x^{0}\right) \delta\left(c^{0}\right) \delta\left(c^{0}\right)$.

$$
\begin{aligned}
& x_{\mu}{ }_{\mu}(\sigma)=x_{\mu}(\sigma)-x_{\mu}(-\sigma) . \\
& c^{0}(\sigma)=c(\sigma)-c(-\sigma) . \\
& \tilde{c}^{e}(\sigma)=\tilde{c}(\sigma)+\tilde{c}(-\sigma) .
\end{aligned}
$$

and $1=c(0) \delta / \delta \tilde{c}(0) \times c(\pi) \delta / \delta \tilde{c}(\pi)$. The ghost number of the factors are $D[0], \delta[1]$ and $1[-4]$. Thus the integral has ghost number -3. It satisfies $\int 0 X=0$ for all $X$ (when the dimension of space-time is 26 ).

\section{The product}

We shall define a product by the expression 


$$
A^{\star} B(3)=\int D_{1} D_{2} K[312] A[1] B(2]
$$

where $D_{1}=\prod_{-i \cdot \pi}^{\pi} d x^{\prime}(\sigma) d c^{\prime}(\sigma) d c^{-1}(\sigma)$. The "naive" kernel $k$ is a product of delta functions which "sew" together half of string A to half of stririg $B$ as shown in rig. 4.

We assume that the integral and " product satisfy the "sewing condition" (eq. 2.24) which implies that $K$ satisfies

$$
\int D_{3} \delta_{3} l_{3} K(312)=\delta[12] \text {. }
$$

The "sewing condition" determines two important properties of $K$ and thus of the "product. It fixes the ghost number of $K$ to be 3 , since $\delta[12]$ has zero ghost number. Thus the "product has ghost number +3 , as desired. It also fixes the kernel $K$ to be Grassman lodd], using $D_{3}$ [even]. $\delta_{3}(00 d), I_{3}$ (even) and $\delta[12]$ leven]. Most of the changes in the axioms can be traced back to the fact that for the closed string the kernel is Grassman lodd), whare as for the open string $K$ is Grassman [even]. To illustrate why this change occurs consider one of the factors, for example D. For the open string $0=\prod_{\sigma=0}^{\pi} d x_{\mu}(\sigma) d c(\sigma) \sigma \tilde{c}(\sigma)$ which in component form is given by $D=\prod_{n=0}^{\infty} \alpha x_{n} \prod_{n=0} \alpha_{n} \sum_{n=1}^{\infty} \alpha \tilde{c}_{n}$. The product $\prod_{n=1}^{\infty} \alpha_{n} \times$. $\partial \tilde{c}_{n}$ is Grassman even; thus the extra zero mode for $c(\sigma)$ makes $D$ Grassman ood. For the closed string $D=\prod_{\sigma-\pi}^{\pi} d x_{\mu}(\sigma) d c(\sigma) v \tilde{c}(\sigma)$ which in component form is given by $D=\prod_{n=-\infty}^{+\infty} \partial x_{n \mu} \prod_{n=-\infty}^{+\infty} d c_{n} \prod_{n=-\infty}^{+\infty} d \tilde{c}_{n}$. Since now thare are an equal number of modes for $c$ and $\tilde{c}, D$ is Grassman even. Similar considerations have been used to obtain the Grassmanality of all the other factors. Since $K$ is Grassman odd, one immediately concludes that A"A is also Grassman nod. 
Let us now reconsider the axioms.

ii) "associativity" -

The expression $\left(A^{n} B\right)^{n} C=-(-1)^{A} A^{n}\left(B^{n} C\right)$ is equivalent to the following familiar pelation for $K: \int D_{3} K(531) K(312]=-\int D_{3} K[513]$ $K[324]$ (see eq.1.31c for the open string). Since $K$ is Grassman ood it is not possible for tise * $t$ (eq.2.27) to be associative for both even and odd Grassman value ids.

The expression $\partial \tilde{c}\left(\partial \tilde{c}\left(A^{*} B\right)^{m} C\right)=\partial \tilde{c}\left(A^{*} \partial \tilde{c}\left(B^{*} C\right)\right)$ is equivalent to the relation for $K$ : $\int D_{3} K_{1}[534] K_{1}[312]=-\int D_{3}^{\prime} K_{1}[513] K_{i}[324]$ where $K_{1}[3 \mid 2]=-\partial_{C_{j}} \tilde{c}_{1} \tilde{c}_{2} K[3 \mid 2]$ and $D=D^{\prime} \partial \tilde{c}$. Note since $K_{1}$ is Grassman even, the above associativity rule does not destinguish between even and odd Grassman valued fields.

iii) $\int A^{* B}=-(-1)^{A B} \int B^{* A}$ This axiom is a direct consequence of the "sewing condition" an' the identity for the closed string $\delta[12]=-\delta\{21]$ where $\delta[12]=\prod_{\sigma=-\pi}^{\pi} \delta_{1}\left(x_{\mu}^{\prime}(\sigma)-x^{2}{ }_{\mu}(-\sigma)\right)$ $\delta\left(c^{\prime}(\sigma)=c^{2}(-\sigma)\right) \delta\left(\tilde{c}^{\prime}(\sigma)+\tilde{c}^{2}(-\sigma)\right)=\prod_{\sigma=0}^{\pi} \delta\left(x^{1}{ }_{e \mu}-x^{2}{ }_{e \mu}\right) \delta\left(x^{1}{ }_{\sigma \mu}+x^{2}{ }_{0 \mu}\right)$ $\delta\left(c_{e}^{1}-c_{e}^{2}\right) \delta\left(c_{0}^{1}-c_{0}^{2}\right) \delta\left(\tilde{c}_{e}^{\prime}+\tilde{c}_{e}^{2}\right) \delta\left(\tilde{c}_{0}^{\prime}-\tilde{c}_{0}^{2}\right)$ and $x_{e}(0)(\sigma)=(\tilde{c}(\sigma)$ $+(-) \times(-\sigma))$. Since there is one additional mode in the expansion of $c_{e}$ .i.e. the zero mode, than there is for $\tilde{c}_{0}$ we obiain the above identity.
v) ine "Lelonitz rule"- $-Q\left(A^{\cdots} B\right)=Q A^{*} B+(-1)^{A} A^{*} O B$ 
Although this rule appears quite unusual it is nevertheless equivalent to the simple condition on $k: \sum_{i=1}^{3} Q_{i} k[312]=0$. Once again the strange sign in this axiom is a direct consequence of the odd Grassmanality of $K$.

\section{Summary and Conclusions}

We have explored the gauge invariant string field theory introduced by witten. We have tried to find a representation of the axioms keeping the anti-commuting coordinates. In particular we were able to find an explicit representation of the integral $\int$ for both the open and closed strings. Unfortunately our representation of the "product was incomplete oue to the special problems associated with the point $\sigma=\pi / 2$ for the open string and $\sigma=0$ and $\pi$ for the closed string. We reel nowever that this technical problem can certainly be overcome [8]. We chose to work with the anti-commuting coordinates in order to be able to use the intuition gained from the component expansions, as described in the text. We believe however that our representation of $\int$ and $*$ is neverthêliess equivalent to the path integral representation discussed by Witten. A proof of this assertion would require an explicit representation of the kernel $K$. One would then be able to explicitly check whether the axioms are satisfied.

In the case of the open string we have assumed that $Q, \int$ and satisfy the requisite axioms. We have then used these axioms to present the BRET invariant action for the interacting string.

In the case of the closed string we have found the free action and 
demonstrated that the equations of motion for the massless sector includes the equations of linearized gravity, as well as the equations for the Kalb-Ramond gauge fie!d and a dilaton. We have then obtained an abstract set of axioms which can be used to construct the gauge invariant action for the interacting closed bosonic string. These axioms, though new, are motivated by properties satisfied by the path integral representation of $\int$ and * discussed by witten. We have assumed that $\int$ has ghost number -3 and * has ghost number +3 . The "sewing condition", which is satisfied by the path integral representation, was used to motivate many of the new axioms. In particular, we used the "sewing condition" to prove the important result that the kernel $K$ for the closed string was Grassman odd. Combining this result with witten's result $\sum_{i=1}^{3} Q_{i} k[3 \mid 2]=0$ we obtain the new "Leibnitz rule". There is one property of the * product which was crucial but not sufficiently motivated. We have assumed that $\partial \tilde{c}\left(A^{*} B\right)=0$ when $\partial \tilde{c} A=\partial \tilde{c} B=0$. This relation is probably satisfied by the path integral representation. The two dimensional manifold on which the * product is defined violates ghost number by +3 . By the Reimann-Roch index theorem there are 3 nontrivial ghost zero modes on this two dimensional surface. Thus $\hat{c}$ and $\overline{\hat{c}}$ are emitted by the manifold. $\tilde{c}$ is contained in $\hat{c}$ and $\overline{\hat{c}}$ and is probably also emitted. As a result we find $\partial_{\tilde{C}}\left(A^{*} B\right)=0$.

If our axioms for the closed string are indeed satisfled by the path integral representation of Witten, we should be able to obtain the scattering amplitudes of the Virasoro-shapiro model using our action. This calculation should be done. 
We are not satisfied with the constraints $\triangle N A=0$ and $A=\partial_{\tilde{c}} X$ which we found necessary to obtain the interacting closed string action. Pernaps these should be thought of as gauge conditions in a theory with an enlarged symmetry group. These constraints are even more unappealing in the context of the closed superstring as discussed by Banks et. al. [13].

Finally, work is now in progress to understand the symmetry principle einbodied in the transformation laws of equations (1.20) for the open string and (2.21) for the closed string. 


\section{Footnotes}

1. General relativity is only valid in the effective low energy theory derined in the zero slope limit.

2. Perhaps a complicated field redefinition of the "graviton" in the Virasoro-Shapiro model does actually transform as $h_{\mu \nu}=g_{\mu \nu}-\eta_{\mu \nu}$ in general relativity-private communication- B. Zwiebach.

3. A similar result for the free closed bosonic string has been obtaines by Banks et. al. [13]. 


\section{References}

1. W. Slegel, Phys. Lett. 1518 . 391; 151B . 396 (1985).

2. W.Siegel ano B. Zwiebach, Nucl. Phys. B263, 105 (1986).

3. E. Witten, "Non-commutative Geometry and String Field Theory", Princeton preprint (1985).

4. T. Banks and M.E. Peskin, Nucl. Phys. B264, 513 (1986).

5. A. Neveu, H. Nicolai, and P. West, Nucl. Phys. B264, 573 (1986); Phys. Lett. 1678, 307 (1986).

6. N. Ohta, Phys. Rev. Lett. 56, 440 (1986).

7. 5.B. Gidolings, "The Veneziano Amplitucie from Interacting String Fielo Theory". Princeton University preprint (1986).

B. N-P Chang, $H-Y$ Guo, $Z$. Qiu and $K$. WU, "Interacting String Field Theory and Chern-Simons Form", City College preprint CCNY-HEP-86/5 (1986).

9. A. Jevicki, "Covariant String Theory Feynman Amplitudes", CERN preprint CERN-TH-4341/85 (1985).

10. H. Aratyn and A.H. Zimerman, "Ghosts and the Physical Modes in the Covarlarit Free string Flelo Theory", Hebrew Unlverslty prepr int RIP-85-9 (1985): "Gauge Invarlance of the uosonic free field String Theory", Hebrew University preprint Print-85-874 (1985).

11. H. Hata, K. Itoh, T. Kugo, H. Kunitomo and K. Ogawa, Manifestly Covariant Field Theory of Interacting string 2.". Kyoto University preprint KUNS-814 (1985); Manifeslly Covariant Field Theory of Interacting String", Kyoto University preprint RIFP-637 (1985). 
12. B. Zwiebach, Gauge Invariant String Actions, MIT preprint MIT-CPT-1308 (1985).

13. T. Banks; M.E. Peskin, C.R. Preltschopr, D. Frledan and E. Martinec, "All Free String Theorles are Theorles of Forms". SLAC preprint SI.AC-PUB-3853 (1985).

14. D. Friedan, Phys. Lett. 162B 102 (1985); "String Field Theory", Chicago University preprint EFI 85-27 (1985).

15. P. Ramond, "A Pedestrian Approach to Covariant String Theory", University of Florida preprint UFTP-85-18 (1985).

16. M. Kaku, "Introduction to the Field Theory of Strings", City College preprint CCNY-HEP-85- 11 (1985); "Supergauge Fleld Theory of Covariant Heterotic Strings", Osaka University preprint OU-HET-79 (1985); Pnys. Lett. 162B, 97 (1985).

17. M. Kaku and J. Lykken, "Supergauge Field Theory of Superstrings", City College preprint Print-85-412 (1985).

18. K. Itoh, T. Kugo, H. Kunitomo and H. Ooguri, "Gauge Invariant Local Action of String field from BRS formalism". Kyoto University preprint KUNS $800 \mathrm{HE}(T H)$ 85/04 (1985).

19. J.-Li: Gervals, "Group Theoretic Approach to the string fleld Theory Action" . Ecole Normale Superleure preprint LPTENS 85/35 (1985).

20. K. Bardakcl, "Covariant Gauge Theory of Strings", Berkeley preprint UCB-PTH-85/33 (1985).

21. S. Raby, R. Slansky and G. West, "Toward a Covarlant String Theory", Los Alamos preprini LA-LR-85-3794 (1985). 
22. A. Cohen, G. Mogre and J. Polchinski, "An Imariant String Propagator". University of Texas preprint UTTG-26-85 (1985); "An Orf-shell Propagator for String Theory". Harvard Unlversity preprint HUTP-85/A058 (1985).

23. A. A. Tseytlin, "Covariant String Field Theory and Effective Action",Lebedev Inst itute preprint LEBEDEV-85-265 (1985).

24. A. Neveu and P.C. West, "The Interacting Gauge Ccuariant Bosonic String", CERN preprint CERN-TH-4315/85 (1985).

25. M.A. Awada. "The Gauge Covariant Formulation of Interacting Strings and Superstrings", Cambridge University preprint Print-85-0937 (1985).

26. D. Pfeffer, P. Ramond and V. Rogers, "GAuge Invariant field Theory of Free Strings", University of Florida preprint UFTP-85-19 (1985).

27. D. Friedan, E. Martinec and S. Shenker, "Conformal Invariance, Supersymmetry and String Theory", Chlcago University preprint Print-86-0024 (1985); Phys. Lett. 160B, 55 (1985).

28. M.E. Peskin and C.B. Thorn, "Equivalence of the Light cone Formulation and the Gauge invariant Formulation of String Dynamics". SLAC preprint SLAC-PUB-3801 (1985).

29. A. Connes, Introouction to Non-Commutative Differential Geometry". "The Chern Character In K Homology", IHES preprints (1982). to appear in IHES Publication.

30. C. Becchi, A. Rovet and R. Stora, Phys. Lett. 52B, 344 (1974); I.V. Tyutin, "Gauge Invariance in Field Theory and in Statistical Mochanics in the Operator Formalism", Lebedev preprint FIAN No. 39 (1975).

31. E.S. Fradk in and G.A. Vilkovisky, Phys. Lett. 55B 224, (1975).

32. I.A. Batalin and G.A. Vilkovisky. Phys. Lett. 898 309 (1977). 
33. T. Kugo and I. Ojima, Phys. Lett. 73B, 459 (1978).

34. J. Schwarz, "Fadeev-Popov Ghosts and BRS Symmetry in String Theories", California Institute of Technology preprint CALT-68-1304 (1985).

35. M. Kato and K. Ogawa, Nucl. Phys. B212 443 (1983).

36. S. Hwang, Phys. Rev. D28, 2614 (1983). 


\section{Figure Captions}

1) This figure illustrates the * operation for open strings. The ' 4 ' half of string $A$ is sewn with the '-' half of string $B$. The remaining halves become the string $A^{*} B$.

2) The integral $S$ sews two halves of the same string. This is illustrated for the string $A^{*} B$. The final result, $S A^{*} B$, could have been obtained by simply sewing strings $A$ and $B$, with opposite orientations, along their entire lengths.

3) The integral $S$ for closed strings also sews two halves of the same string. This results in two singular points, labelled $\sigma=0$ and $\sigma=\pi$ in the figure.

4) This figure lllustrates the * operation for closed strings. Half of string $A$ is sewn with half of string $B$. The remalning halves become the closed string $A * B$ 
(I)

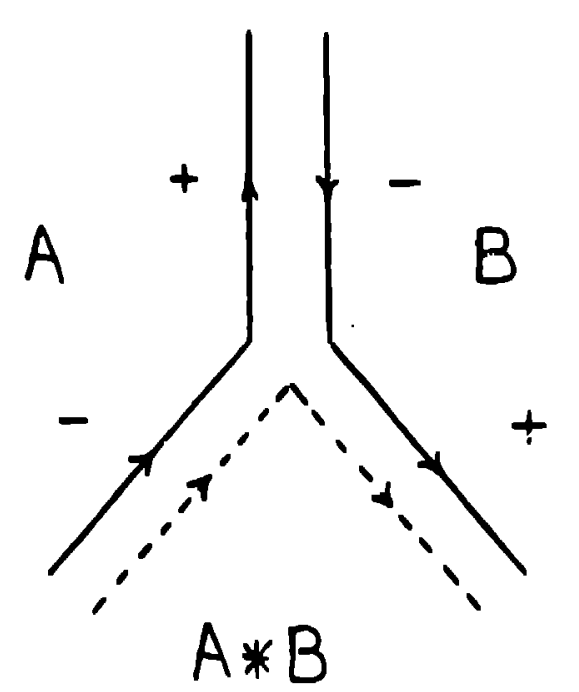

(2)

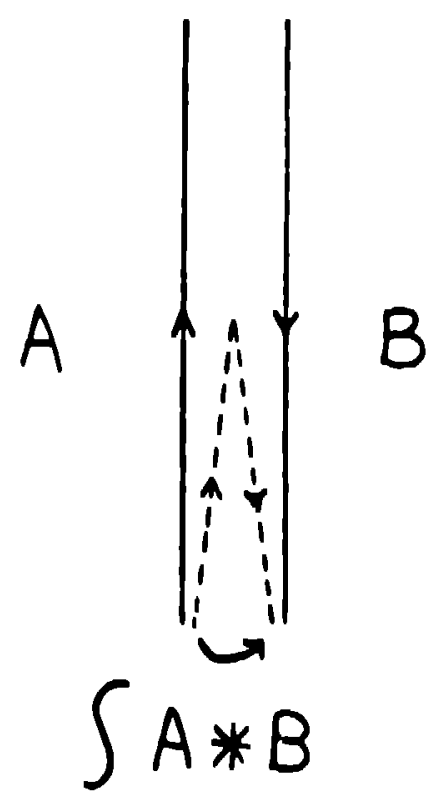


(3)

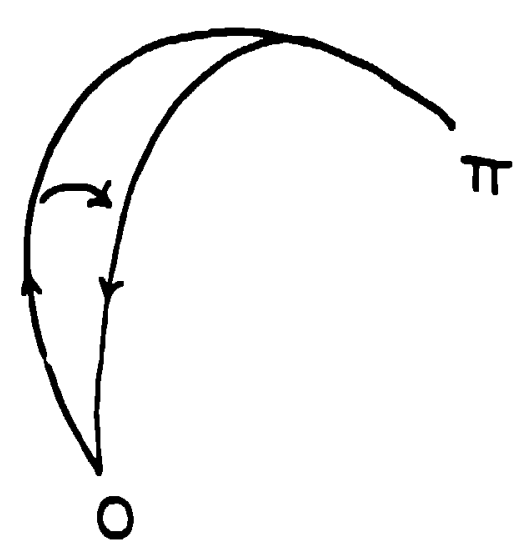

(4)

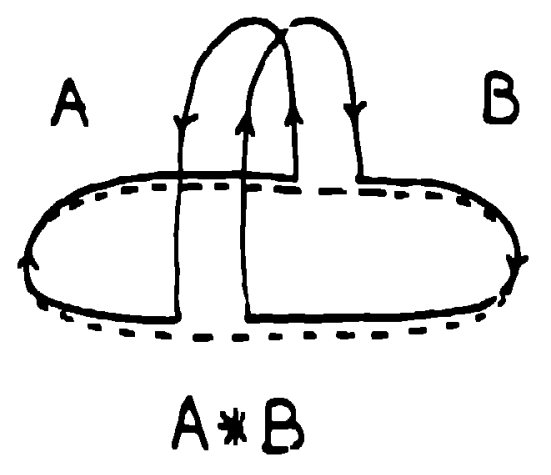

